\title{
Tracking the Fate of Surface Plasmon Resonance- Generated Hot Electrons by In Situ SERS Surveying of Catalyzed Reaction
}

\author{
Rui Liu, Zuoliang He, Jiefang Sun, Jingfu Liu, * and Guibin Jiang
}

\begin{abstract}
Plasmonic catalysis is an emerging process that utilizes surface plasmon resonance $(S P R)$ process to harnesses solar energy for the promotion of catalyzed reactions. In most cases, SPR generated hot electrons (HEs) play an indispensable role in this solar-chemical energy shift process. Therefore, understanding the effectiveness of the HEs in promoting chemical reactions, and identifying the key factors that contribute to this utilization efficiency is of profound importance. Herein, the authors outline an in situ surface enhanced Raman spectroscopy protocol to track the fate of HEs. This is based on the unheeded HEs-acceleration nature of the p-nitirothiophenol hydrogenation reaction. By this way, the authors discover that unlike Au@Pd nanostructures which experience a 20-fold increase in rate constant, HEs primary leak to surrounding $H^{+} / O$ species through Ag pinholes in Ag@Pd. This work sheds light on why Ag is seldom employed as a plasmonic cocatalyst, and provides a new viewpoint to design plasmonic nanocatalysts with efficient light utilization.
\end{abstract}

\section{Introduction}

Solar light is the ultimate energy source for the whole biosphere. ${ }^{[1]}$ With the increasing demand for clean and sustainable energy supply, the low energy conversion efficiency of photosynthesis, ${ }^{[2]}$ and the imminent exhaustion of fossil fuels, the development of efficient alternative pathways to harvest solar energy has become a worldwide priority. ${ }^{[3]}$ From this regard, noble metal nanoarchitectures gained significant

Dr. R. Liu, Z. L. He, Dr. J. F. Sun, Prof. J. F. Liu, Prof. G. B. Jiang

State Key Laboratory of Environmental Chemistry and Ecotoxicology

Research Center for Eco-Environmental Sciences Chinese Academy of Sciences

Beijing 100085, China

E-mail: jfliu@rcees.ac.cn

Z. L. He

College of Resources and Environment University of Chinese Academy of Sciences

Beijing 100049, China

DOI: $10.1002 /$ smll.201601773 attention for their ability to capture and store solar energy in the form of chemical energy. ${ }^{[4]}$ The material base for this is the coherent oscillation of the surface free-like electrons under the excitation of resonance photon, a phenomenon known as surface plasmon resonance (SPR). ${ }^{[4 b, 5]}$ SPR active noble metals nanoparticles (NPs) absorb/scatter light with a molar extinction coefficient of $10^{9} \mathrm{M}^{-1} \mathrm{~cm}^{-1}$, ${ }^{6]}$ which is 3 orders of magnitude higher than that of traditional organic dyes $\left(<10^{6} \mathrm{M}^{-1} \mathrm{~cm}^{-1}\right)$. This strong light-matter interaction results in a temporary confinement of a huge amount of energy ${ }^{[4 b]}$ and has been widely studied as a potential source of light and heat. ${ }^{[7]}$ Moreover, through a Landau/chemical interface damping process, the captured energy is capable of directly depositing into a surface adsorbate (e.g., reactant/intermediate) in the form of hot electrons (HEs). This energy input alters the energy diagram of the reaction and has been shown to enhance the rate of many chemical reactions such as hydrocarbon conversion, ${ }^{[7 c, 8]}$ small molecule oxidation, ${ }^{[9]}$ hydrogen evolution, ${ }^{[10]}$ carbon-carbon coupling, etc., ${ }^{[11]}$ and fulfill the solar energy/chemical energy shift process. Meanwhile, these systems have also been studied from the viewpoint of catalyzing reactions. Compared with other energy input routines, the above SPR-generated HEs can 
Matails

www.MaterialsViews.com

be precisely deposited into certain acceptor states (e.g., the antibonding orbital of $\mathrm{H}_{2} / \mathrm{O}_{2}$ ) and efficiently facilitates the activation of target molecule. ${ }^{[9,12]}$ This gentle and effective energy input is unique and important because it increases the longevity of reactive centers and suppresses the side reactions. ${ }^{[13]}$

Although HEs have been demonstrated to be effective in promoting chemical reactions, there are cases where they were generated but were not accurately deposited into the desired channels. Especially when multiple processes take place during the reaction, various surface adsorbate/reactants, including $\mathrm{H}^{+}$and $\mathrm{O}_{2},{ }^{[5 a, 14]}$ compete for the limited HEs. This, on one hand, makes the direct activation of $\mathrm{H}^{+}$and molecular $\mathrm{O}_{2}$ as reductant/oxidant feasible in a series of reactions. ${ }^{[5 a, 9]}$ On the other hand, the produced HEs are at high risk of being wasted if they are meant to be used in other chemistry. A sophisticated design of SPR-active catalysis and reaction setup is therefore required to achieve a highly efficient capture/utilization of sunlight. From this point of view, we have a special interest in Ag-based plasmonic catalysts. Compared to $\mathrm{Au}, \mathrm{Ag}$ is 100 times less expensive and also has 10 times stronger photon/energy capture capacity. ${ }^{[15]}$ Intriguingly, there have only been a few reports using Ag as a plasmonic cocatalyst, and limited to the oxidation of small gaseous molecules $^{[9]}$ and the formation of a Ag-semiconductor Schottky barrier. ${ }^{[16]}$ The physical-chemical factor that would explain the limited cooperation between Ag and transit metal catalysts during the light-chemical energy shift process, and how to get rid of this factor, are still unknown.

Herein employing the catalyzed reduction of p-Nitrothiphenol (p-NTP) as a probe reaction, ${ }^{[17]}$ we explore the first example of tracking the fate of SPR generated-HEs. Although have being studied for more than two decades, and frequently utilized as demo reaction for SERS ${ }^{[17 \mathrm{~d}, \mathrm{~g}]}$ and tip enhanced Raman scattering (TERS) ${ }^{[18]}$ surveying of catalyzed reactions for kinetics and mechanistic study, one open question is whether the SPR property of the SERS active nanostructures, which is mean to amplification the Raman signal only, has other profound effect on the reaction itself. In this work, by interpreting the relationship between the laser power density/wavelength and the rate constant of Au@Pd ultrathin nanowires (NWs) catalyzed reduction of p-NPT, we confirmed the light-enhancement effect in this reaction, and correlated it to the SPR generated-HEs from Au ultrathin NWs. Then, we utilize this process to test whether the SPRgenerated HEs from $\mathrm{Ag}$ atoms are available to the $\mathrm{Pd}$ atoms and accelerate the reduction reaction of p-NTP on Ag@Pd nanoporous film (NPF). Finally, based on the "finger print" spectra provision capacity of SERS, the factors that influence the fate and the utilization efficiency of HEs were figured out.

\section{Results}

\subsection{Kinetics Characteristics of p-NTP Reduction Reaction under Light}

The influence of SPR-generated HEs to the reduction of p-NTP was probed by SERS studying the laser power density-rate constant relationship throughout the reaction. For doing so, we deposited an Au@Pd (with a Pd coverage of $1 / 4$ monolayer, ML) NWs thin films onto a transparent $\mathrm{SiO}_{2}$ slide $(250 \mu \mathrm{m}$ thick). The as-prepared film, whose field emission scanning electron microscopy (FESEM) image is presented in Figure 1A and Figure S1 in the Supporting Information, shows both highly reproducible SERS activity (Figure 1C, and blue dots in 1D) and high catalytic activity (Figure S2, Supporting Information). Moreover, the ultrathin diameter of $\mathrm{Au} @ \mathrm{Pd}$ NWs $(\approx 3 \mathrm{~nm})$ pledges effective generation of the HEs. ${ }^{[12]}$ The experiment setup and details are given in Figure S3 in the Supporting Information. The representative $3 \mathrm{D}$ reaction trace presented in Figure S2 in the Supporting Information shows that, upon the addition of $0.2 \times 10^{-3} \mathrm{M}$ ice-cold $\mathrm{KBH}_{4}$ solution, the Raman band at $1340 \mathrm{~cm}^{-1}\left(v_{\mathrm{NO}}\right.$ of the $\left.-\mathrm{NO}_{2}\right)$ attenuates immediately and almost completely disappear after $20 \mathrm{~s}$. This is followed by the appearance of a new Raman band at $1592 \mathrm{~cm}^{-1}$, indicating the conversion of p-NTP to p-aminobenzenethiol (p-ABT) (Figure S4, Supporting Information). No peaks at $1140,1388,1430 \mathrm{~cm}^{-1}$ were identified at the current time resolution $(\approx 0.1 \mathrm{~s})$, which suggests no 4,4'-demercaptoazobenzene (DMAB) generated during the reaction. Kinetics analysis revealed that the reaction is first-ordered with a rate constant of $0.13 \mathrm{~s}^{-1}$. Furthermore, the reduction took place very fast and no induction time was observed. This implies that catalyzed by $\mathrm{Pd}$ atoms on $\mathrm{Au} \mathrm{NWs}, \mathrm{KBH}_{4}$ molecules efficiently reduce the $\mathrm{p}$-NTP. These results match well with the previous results. ${ }^{[17 a, 19]}$

Since the SERS spectra are collected over a very small area, point to point Raman linescan was performed from -60 to $60 \mu \mathrm{m}$ with a stepsize of $3.0 \mu \mathrm{m}$ to clarify whether the collected spectra are representative enough (which is the precondition for in situ SERS surveying of catalyzed reactions), and to inspect the reaction progress of the nearby area (Figure 2A and Figure S5, Supporting Information). Surprisingly, the disappearance of the Raman peak at $1340 \mathrm{~cm}^{-1}$ is localized at the origin point. At increasing distances from the laser center, the intensity of the peak increases exponentially. When the distance is larger than $9 \mu \mathrm{m}$, there is almost no attenuation of the intensity of $-\mathrm{NO}_{2}$ and no peak for $-\mathrm{NH}_{2}\left(1590 \mathrm{~cm}^{-1}\right)$ is observed (Figure 2B). Considering the highly uniformity of the Au@Pd NWs, and the even distribution of catalytically active $\mathrm{Pd}$ atoms, the above valley shape reaction progress suggests that the reaction is asynchronous. The disappearance of peaks corresponding to p-NTP in successive SERS observation only suggests that the reaction goes to completion at the illuminated area. More importantly, the width of the valley is $\approx 20 \mu \mathrm{m}$ (Figure $2 \mathrm{~A}$ ), which is similar in size to the laser spot diameter. This indicates that the reaction is confined to the illuminated area. If this theory holds, since the intensity distribution of the laser follows the Poisson/Gauss rule, the peak area for the Raman band at $1340 \mathrm{~cm}^{-1}$ along the scanned line will take the form of a Poisson/Gaussian curve. As is shown in Figure 2C, the experiment data (blue dots) is in line with the fitted values (red line). This unambiguously confirms that laser illumination accelerates this reaction and that the degree of acceleration is proportional to the laser power density. 


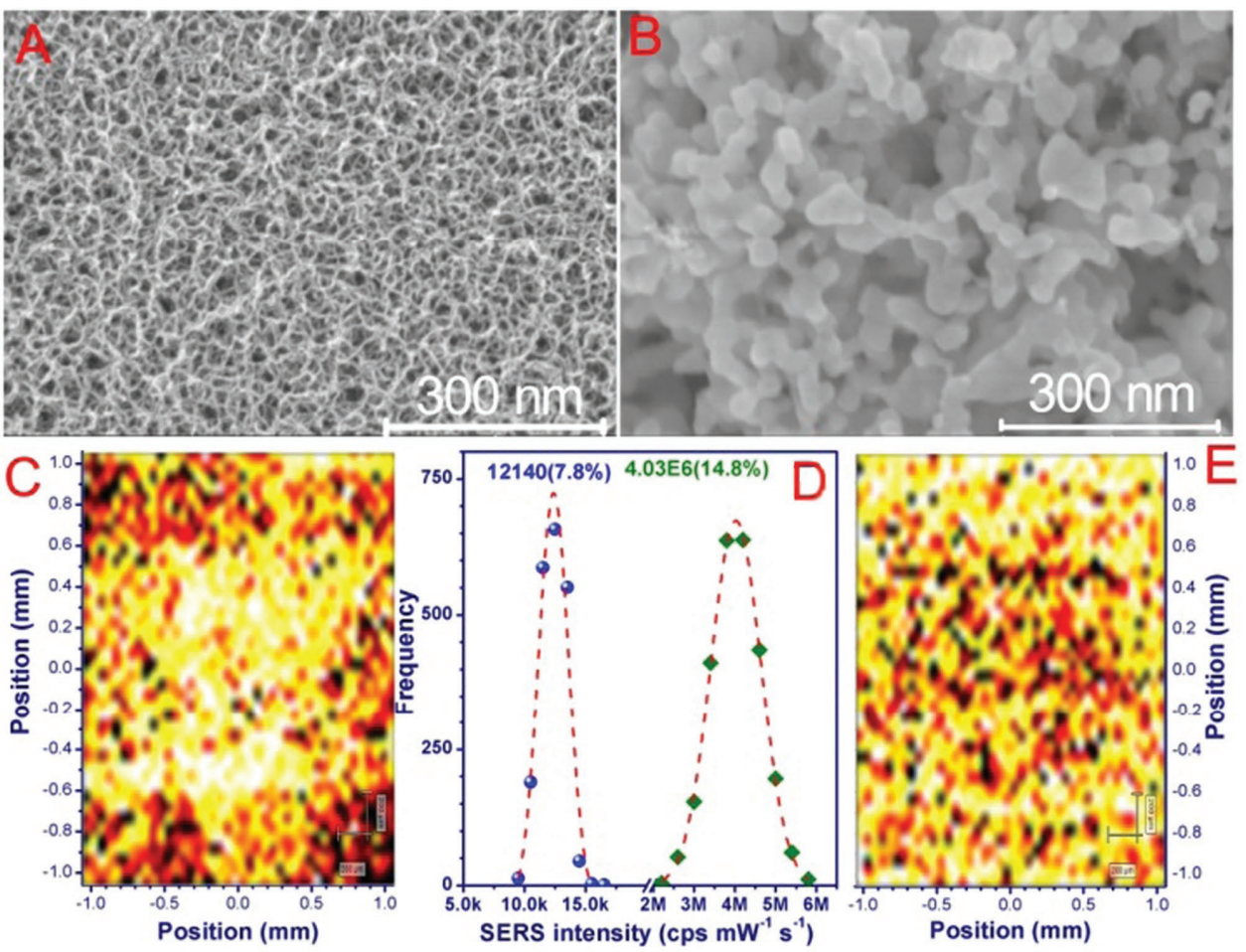

Figure 1. The morphology and the SERS performance of Au@Pd NWs film and Ag@Pd NPF utilized in this study. FESEM image A), large area (2 mm $\times$ $2 \mathrm{~mm}$ ) SERS mapping C) and the corresponding SERS intensity distribution of the Raman band at $1340 \mathrm{~cm}^{-1} \mathrm{D}$, left) of Au@Pd NWs film and that of Ag@Pd NPF B,E,D, right).

In situ SERS monitoring over changing laser power densities was conducted to further demonstrate the SPRenhancing nature of this reaction. Figure $\mathbf{3 A}$ displays the
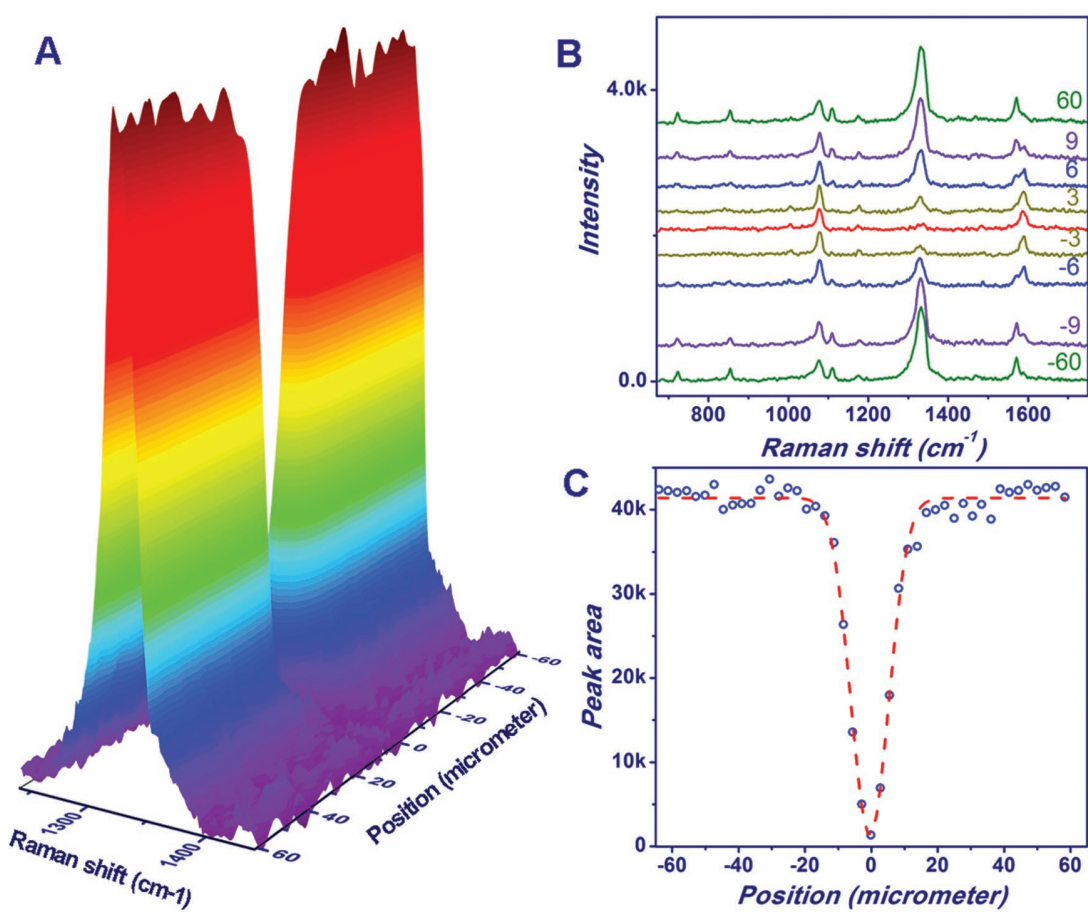

Figure 2. Linescan SERS spectra shows the SPR acceleration effect during the catalyzed reduction of p-NPT. A 3D Raman map of the band at $1340 \mathrm{~cm}^{-1} \mathrm{~A}$ ), representative SERS spectra taken at different positions B), and a Poisson/Gaussian distribution plot generated by the change in intensity of the Raman band at $1340 \mathrm{~cm}^{-1}$ versus position C). kinetics data excited by a $785 \mathrm{~nm}$ laser as the power density varied from $2.5 \times 10^{3}$ to $2.5 \times 10^{5} \mathrm{~mW} \mathrm{~cm}^{-2}$. When the reaction is excited by a high density laser $\left(\approx 10^{5} \mathrm{~mW} \mathrm{~cm}^{-2}\right.$, pink and dark red dots), it occurs immediately. However, when the laser power was lowered to $2.5 \times 10^{4} \mathrm{~mW} \mathrm{~cm} \mathrm{~cm}^{-2}$, an induction period of $\approx 30 \mathrm{~s}$ was observed, and during this time, there was no significant peak intensity decline. This induction period increased to $\approx 200 \mathrm{~s}$ with a laser power density of $5.0 \times 10^{3} \mathrm{~mW} \mathrm{~cm}^{-2}$. In all cases, after the induction time, first-ordered reductions of p-NTP to p-ABT were observed. The gradual elongation of induction time was accompanied by a lowered slope of the kinetics curve as the laser power density decreased. This supports our assumption that light does accelerate/promote this reaction, approximately, through photothermal (PT) effect and HEs.

\subsection{Mimicking the "Dark Reaction" by Place-Shift SERS}

Up to now, we have demonstrated that during the SERS surveying of the catalyzed reduction of p-NTP by $\mathrm{KBH}_{4}$, besides as excitation source for the SERS spectra, the continuous laser illumination significantly accelerates the reaction itself. 


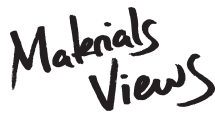

www.MaterialsViews.com
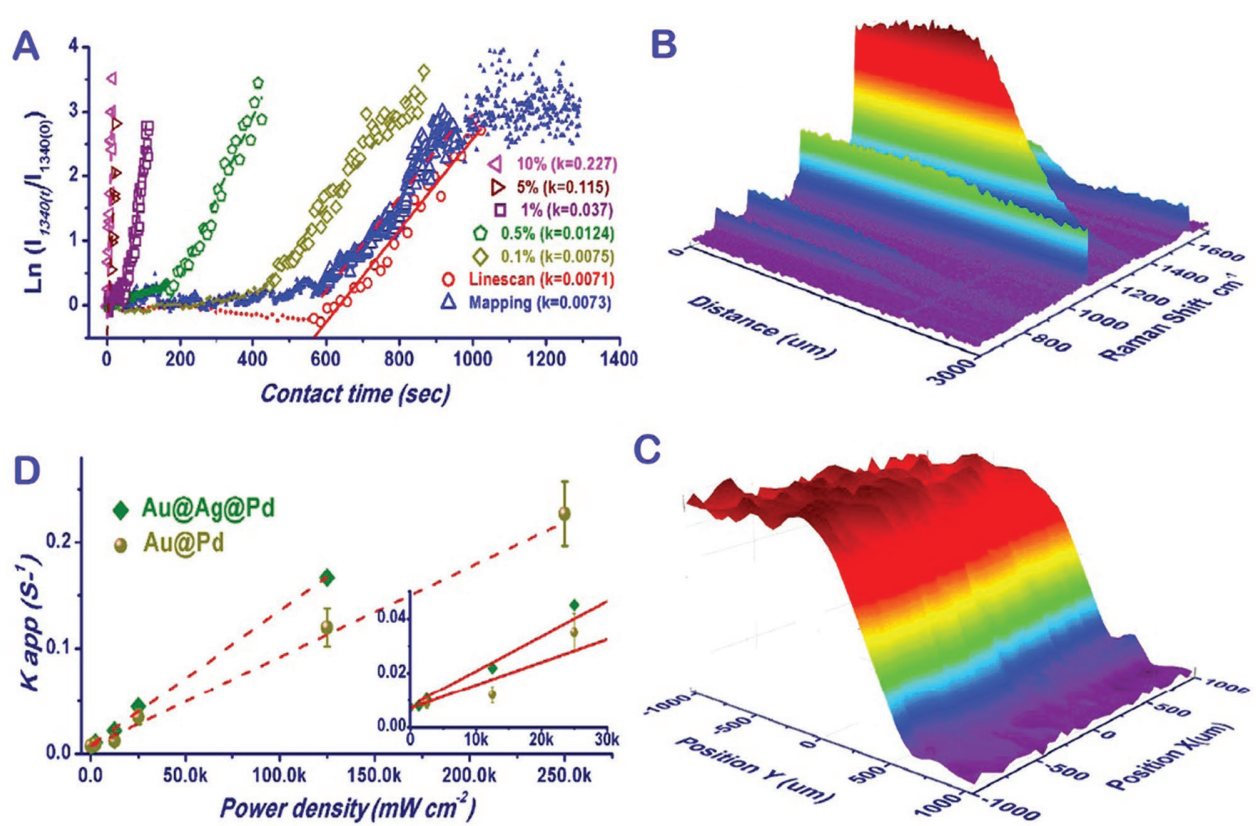

Figure 3. Kinetic characteristics of p-NTP reduction on Au@Pd NWs film derived from in situ SERS study at different power density A), mimic the dark reaction by linescan and Raman mapping $B, C$ ), the reaction rate as a function of power density D).

Then, one may naturally wonder whether this reaction occurs in the absence of light or whether Pd atoms are the real catalytic center, and what is the physical-chemical nature behind this unprecedented phenomenon. Meanwhile, now that the observed reaction has been accelerated already, is in situ SERS study still applicable for reaction monitoring, especially for kinetic study? And how can we get rid of this artificial light effect? To answer these questions, we further lowered the relative laser power to $2.5 \times 10^{3} \mathrm{~mW} \mathrm{~cm}^{-2}$ (yellow trace in Figure $3 \mathrm{~A}$ ), where the induction time further increased to $\approx 500 \mathrm{~s}$, and the rate constant slightly decreased from 0.012 to $0.0085 \mathrm{~s}^{-1}$. However, this is still not safe to conclude that the plasmonic enhancement effect has been completely suppressed. Because of the moderate SERS enhancement factor of Au@Pd NW film ( $\approx 10^{3}-10^{4}$, Figures S6 and S7, Supporting Information), lowering the laser power further is challenging. Therefore, to successfully mimic the "dark reaction," we use what we know about the large area uniformity of the Au@Pd NW film, and the high localized nature of the light acceleration that has been demonstrated in Figure 2A. Raman mapping or linescanning over a large area using a step-size larger than the spot size of laser, which we termed as place-shift SERS, was performed. By doing this, not only the illumination time on each sample point was shortened, but also the light acceleration effect can be offset. Figure 3B shows the $3 \mathrm{D}$ reaction trace recorded through scan along a $3 \mathrm{~mm}$ line with a stepsize of $20 \mu \mathrm{m}$ (150 spectra, $8 \mathrm{~s} / \mathrm{spectra}$, red dots in Figure 3A). The induction stage lasted for $600 \mathrm{~s}$ and was followed by a gradual reduction of the peak at $1340 \mathrm{~cm}^{-1}$ until the reaction was complete. The kinetic analysis reveals that the reaction derived from linescan is also first-ordered. Compared with the case using a $2.5 \times 10^{3} \mathrm{~mW} \mathrm{~cm}^{-2}$ laser, there is a longer induction time and a slightly decreased rate constant of 0.0075. Raman mapping was also performed on a $2.0 \mathrm{~mm} \times$ $2.0 \mathrm{~mm}$ area with a step size of $50 \mu \mathrm{m} \times 50 \mu \mathrm{m}$ (Figure $3 \mathrm{C}$,
1681 spectra, $0.45 \mathrm{~s} /$ spectra) during the reaction. The three stages of this reaction (i.e., the induction stage, the reduction stage, and the final stage) were clearly marked as a plateau, a steep slope and a ground, respectively. Coincidently, although different laser densities were intentionally selected $\left(2.5 \times 10^{3}\right.$ and $1.25 \times 10^{5} \mathrm{~mW} \mathrm{~cm}^{-2}$ for linescan and mapping, respectively), the induction time and the rate constant that were calculated from linescan and mapping are almost identical. This consistency strongly supports the validation of the proposed place-shift strategy (Raman mapping or linescan) in suppressing the photoacceleration effect. Meanwhile, the rate constant is still larger than the reported data of Pd NPs, ${ }^{[17 b]}$ and high facet indexed $\mathrm{Au}$ nanosphere, ${ }^{[20]}$ confirms the high catalytic activity of Pd atoms on Au NWs.

\section{3. p-NTP Reduction Reaction as an Ideal Process to Track the Fate of HEs}

Upon consolidating the photoacceleration nature of $\mathrm{p}-\mathrm{NTP}$ reduction process, and handled the interference from the in situ SERS study of the reaction kinetics data, we could then answer how the reaction was accelerated by the light. Figure 3D summarizes the relationship between the laser power density and apparent rate constant. It shows that there is a linear dependence of the apparent rate with the source intensity, and that, as the laser density was increased from $2.5 \times 10^{3}$ to $2.5 \times 10^{5} \mathrm{~mW} \mathrm{~cm}^{-2}$, a 25 -fold enhancement in rate constant was observed. Moreover, the high linearity of 0.96 between the power density and the rate constant indicates that the SPR-generated HEs were responsible for the observed photoactivity. ${ }^{[4 a]}$

Besides the apparent rate constant dependence on power density, the reaction can also be modified by strengthened/ weakened light-matter interactions. For example, due to 
the ultrathin nature of the Au@Pd NWs, and the interband transition between the Au core and Pd shell, Au@Pd NWs displayed limited light adsorption. However, this can be partially alleviated by introducing an Ag buffer layer between Au and Pd (Au@Ag@Pd NWs, unpublished result, Figure S8, Supporting Information). Indeed, as is presented in Figure 3D (green dots), with the laser power density increase from $2.5 \times 10^{3}$ to $1.25 \times 10^{5} \mathrm{~mW} \mathrm{~cm}^{-2}$, the rate constant linearly increased by 21 times from 0.008 to $0.167 \mathrm{~s}^{-1}$. On the other hand, when switched to $633 / 532 \mathrm{~nm}$ laser (i.e., in the SPR "off" region), even in the presence of a $1.0 \mathrm{~mW}$ laser that corresponds to a power density of $10^{5}-10^{6} \mathrm{~mW} \mathrm{~cm}^{-2}$, no photo acceleration was observed on Au@Pd NWs (Figure S9, Supporting Information).

The above data collectively demonstrate that during the in situ SERS surveying catalyzed reduction of p-NTP with $\mathrm{KBH}_{4}$, the observed reaction probably already has been accelerated by SPR-generated HEs. Currently, how HEs take part in this process is still under investigation, but facilitating the activation of $-\mathrm{NO}_{2}$ is the most likely pathway (see Discussion). Meanwhile, the photoacceleration phenomenon is constrained to the laser illuminated area, this is ascribed to the short lifetime of HEs. ${ }^{[4 b]}$ Anyway, this enables us to monitor the intrinsic rate constant by in situ SERS using a low power density laser, or through Raman mapping/ linescan over a larger area. Moreover, the catalyzed reduction of p-NTP is an ideal demo reaction to track the fate of HEs. For instance, the length of the induction time during the reaction is a reliable indicator of the generation of HEs, while the kinetic data derived from the quantitative SERS can be used to evaluate the degree of enhancement in the reaction. Furthermore, the fingerprint-spectra provision capacity of SERS shed light on how the HEs wasted/used.

\subsection{HEs Transfer Behaviors in Ag@Pd}

As a proof-of-concept demonstration, we investigate the fate of Ag-generated HEs by using the developed protocol, where a Pd layer was again deposited on $\mathrm{Ag}$ surface to make $\mathrm{Ag}$ catalytically active (Figure $1 \mathrm{~B}) .{ }^{[21]}$ The enhanced light-matter interaction in Ag@Pd is illustrated in Figure 1D,E, where a significantly lower density of p-NPT molecules generated 400 times higher normalized SERS intensity than that of Au@Pd (see Discussion in the Supporting Information). This enables the SERS monitoring of the reaction with a power density $\left(5 \mathrm{~mW} \mathrm{~cm} \mathrm{~cm}^{-2}\right.$ or $\left.0.1 \mu \mathrm{W}\right)$ that is comparable to or lower than that of sunlight $\left(\approx 100 \mathrm{~mW} \mathrm{~cm}^{-2}\right) .{ }^{[9]}$ Similar to the reactions using the Au@Pd NWs film, after an induction time, the Raman band associated with $-\mathrm{NO}_{2}$ gradually decreases with a first-ordered rate constant of $0.0092 \mathrm{~s}^{-1}$. However, the induction time was only $\approx 70 \mathrm{~s}$, which is much shorter than that on Au@Pd NWs film. This was ascribed to the expected enhanced light-mater interaction. When the power density was simply increased to $2.5 \times 10^{3} \mathrm{~mW} \mathrm{~cm}^{-2}$ or above, no induction period was observed, and the reduction of $\mathrm{p}-\mathrm{NTP}$ occurred immediately. This again demonstrates the induction time is determined by the laser power, and the enhanced light-matter interaction result in short induction time.
However, after a detailed kinetic analysis that summarized in Figure 4A,B, it was noteworthy that the reduction process for p-NTP on Ag@Pd cannot be simply described by the firstorder model. The data showed a gradual increase in the slope and finally resulted in a steady rate constant. This trend was observed at different laser powers $\left(5.0-5.0 \times 10^{4} \mathrm{~mW} \mathrm{~cm}^{-2}\right)$ and different wavelengths $(785,633$, and $532 \mathrm{~nm})$. Moreover, as illustrated in Figure $4 \mathrm{~B}$, the statistical analysis revealed that the steady rate constants from each case have insignificant difference. They are also comparable to the rate constants derived from linescan. Therefore, laser has a negligible effect on the reaction rate of p-NTP on the Ag@Pd system. Next, we explored why there was no apparent photo acceleration process on Ag@Pd surface and where the generated HEs were ultimately reacted.

Upon a closer inspection of the reaction trace from the continuous SERS spectra, we found that DMAB, a typical product from the oxidation of $\mathrm{p}$-ABT by HEs-activated ${ }^{3} \mathrm{O}_{2},{ }^{[14 a]}$ or the HEs induced dimerization of $\mathrm{p}$-NTP that first reported by Sun et al., ${ }^{[22]}$ was generated immediately after the addition of $\mathrm{KBH}_{4}$ (Figure S8, Supporting Information). Moreover, the generated DMAB is in the trans-configuration, ${ }^{[22 b]}$ and its amount is dependent on the power density and the wavelength of the laser. At low power density $\left(5 \mathrm{~mW} \mathrm{~cm}^{-2}\right)$ or during linescan, there are only trace amounts of DMAB, with increasing laser power density, using a 532/633 nm laser, the relative amount of DMAB largely increased (Figure 4C and Figure S9, Supporting Information). The above facts confirm the effective generation of HEs on Ag@Pd. On the other hand, the evolution of the relative amount of DMAB is power density/wavelength independent. Over the course of the reaction, there is a quick increase in DMAB amount during the first few seconds, followed by an unchanged amount in the next $\approx 100 \mathrm{~s}$, and then an exponentially decay. Coincidently, higher $\mathrm{p}$-NTP reduction rates were accompanied by lower amounts of DMAB. Also, after the amount of DMAB decreased to less than $10 \%$ of its original value, the rate constant remained unchanged. We hypothesize that these DMAB, at least a large portion, are from the oxidation of p-ABT via the route reported by Huang et al. (Figure S10, Supporting Information). ${ }^{[14 a]}$ The relative amount of DMAB is controlled by the balance between DMAB generation (amount of p-ABT and active surface O species) and its dissociation, either through its back-reduction to p-ABT by active $\mathrm{H}$ or the direct break of the $-\mathrm{N}=\mathrm{N}-$ bond with $\mathrm{HEs}$ and formation of $\mathrm{SC}_{6} \mathrm{H}_{5} \mathrm{~N}$ radical fragment. ${ }^{[23]}$ Therefore, the disappearance of DMAB is associated with the stepwisely elimination of surface $\mathrm{O}$ species either via $\mathrm{p}$-ABT consumption or reduction by $\mathrm{KBH}_{4}$. To affirm this hypothesis, we evaluated the dependence of DMAB disappear time on the $\mathrm{KBH}_{4}$ concentration (Figure 4D). With increasing $\mathrm{KBH}_{4}$ concentration, both the relative amount of DMAB generated and the start time for its attenuation decreased simultaneously. Meanwhile, the rate constant for the p-NTP reduction increased linearly with increasing $\mathrm{KBH}_{4}$ concentration. This suggests that the presence of DMAB is associated with the back oxidation of p-ABT. As the concentration of the reactive $\mathrm{H}$ species on the Ag@Pd surface is limited, the formation of DMAB, and thus its back reduction to $\mathrm{p}-\mathrm{ABT}$ leads 


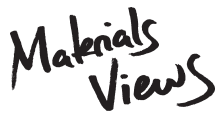

www.MaterialsViews.com
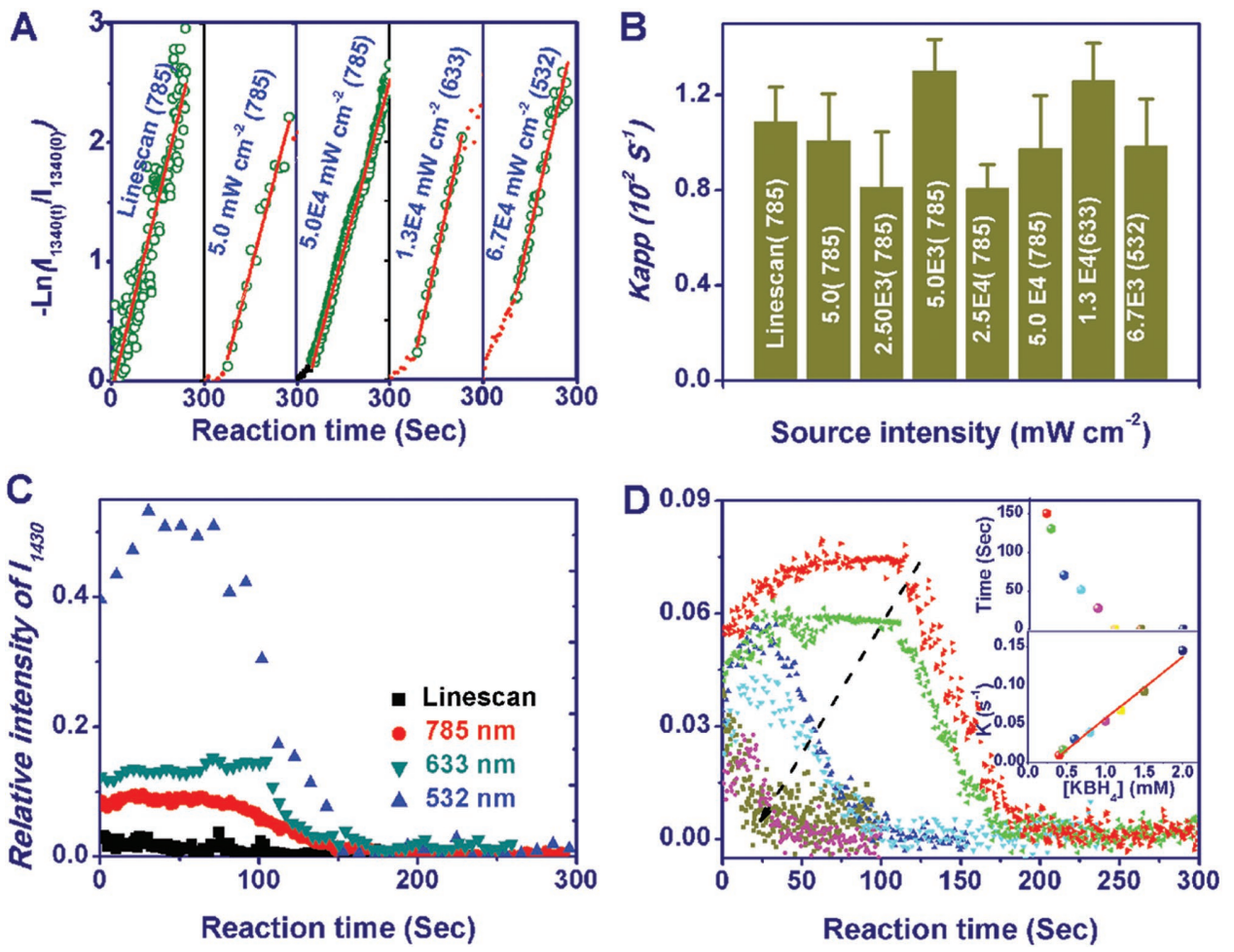

Figure 4. Effect of the laser power density and wavelength on the reduction pace of p-NTP on Ag@Pd (Pd coverage is 0.5\%) A,B). The evolution of the amount of DMAB in different laser wavelength $\mathrm{C}$ ) and different $\mathrm{KBH}_{4}$ concentration $\mathrm{D}$ ). Inset in (D), the time for DMAB disappearance and the rate constant in different $\mathrm{KBH}_{4}$ concentration.

to a waste of active $\mathrm{H}$ species. This leads to a lower p-NTP reduction rate during the beginning of the reaction. Moreover, besides consumes active $\mathrm{H}$ species, the sensitization of $\mathrm{O}$ into ${ }^{1} \mathrm{O}_{2}$ also needs HEs, ${ }^{[14 a]}$ and both facts reflected in the low reaction rate. Meanwhile, during the linescan process, the amount of HE-generated active $\mathrm{O}$ species is reduced, which led to low amounts of DMAB. This also supports that DMAB is generated via the oxidation of p-ABT.

\subsection{Blockage of Ag Pinhole for Efficient Utilization of HEs in Ag Based Plasmonic Catalyst}

However, this still cannot fully explain why HEs does not promote the p-NTP reduction on Ag@Pd. Other pathways must consume the produced HEs, especially after the removal of $\mathrm{O}$ species by $\mathrm{KBH}_{4}$ (disappearing of DMAB). Considering the existence of a large amount of $\mathrm{H}^{+}$in the aqueous phase, the most plausible pathway for HEs is to react with $\mathrm{H}^{+},{ }^{[17 \mathrm{e}]}$ a process similar to the hydrogen evolution process. Traditionally, with the excitation/donation of HEs, the gradual accumulation of holes $\left(\mathrm{Ag}^{+}\right)$on the surface of $\mathrm{Ag}$ nanostructures results in the termination of recruiting HEs. However, Xie et al. recently proposed a photocatalytic cycle that involves the formation of low solubility photoactive $\mathrm{AgX} .{ }^{[5 \mathrm{a}]}$ This results in the photoreduction of $\mathrm{Ag}^{+}$and enables the continuous activation of $\mathrm{H}^{+}$. Moreover, this transforms the previously inactive Ag into an active catalytic species for the reduction of $\mathrm{p}$-NTP. In our case, a similar pathway may be also present. The reduction environment offered by
$\mathrm{KBH}_{4}$ is capable of reducing $\mathrm{Ag}^{+}$and running the cycle, and is expected to continuous product and waste of HEs. Therefore, it is plausible that, although part of the HEs from $\mathrm{Ag}$ are transferred to the surface $\mathrm{Pd}$ atoms, which activate $\mathrm{KBH}_{4}$ into active $\mathrm{H}$ species and reduce $\mathrm{p}$-NTP into $\mathrm{p}$-ABT, the majority of the HEs are consumed directly by $\mathrm{H}^{+} / \mathrm{O}$ species. At the same time, the presence of $\mathrm{KBH}_{4}$ can back-reduce the $\mathrm{Ag}^{+}$species into $\mathrm{Ag}^{0}$ species. In other words, the presence of $\mathrm{Ag}$ pinholes in the $\mathrm{Ag} @ \mathrm{Pd}$ nanoarchitecture is responsible for the wasting of HEs (Figure S12, Supporting Information). It should be noted that this process may be influenced by the

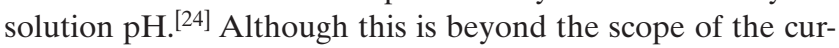
rent study, it deserved to be discussed in future works.

To further validate that the HEs are self-consumed in the Ag surface, and therefore hinders the application of Ag@Pd as plasmonic catalyst, we plug the $\mathrm{Ag}$ pinholes by increasing the Pd coverage to $4 \%$ (Figure S11, Supporting Information). Although this resulted in the formation of a trace amount of $\mathrm{Pd}$ NWs on the Ag surface and lowered the SERS activity of the material (Figure S12, Supporting Information), the amount of surface Ag atoms was largely decreased. Figure S13 in the Supporting Information displays that no DMAB was observed over the whole power density range for $785 \mathrm{~nm}$ laser from $2.5 \times 10^{3}$ to $5.0 \times 10^{5} \mathrm{~mW} \mathrm{~cm}^{-2}$. This suggests that $\mathrm{Ag}$ oxides/hydroxides are not present and that there is a negligible reaction between $\mathrm{HEs}$ and $\mathrm{O}$ spices. Consequently, the rate constant was increased by 4 -fold from 0.026 to $0.105 \mathrm{~s}^{-1}$ (Figure 5A). It is deserved to point out that maybe for the partial damped Ag SPR activity by Pd overlayer, an induce time was observed. A similar trend was observed when a 

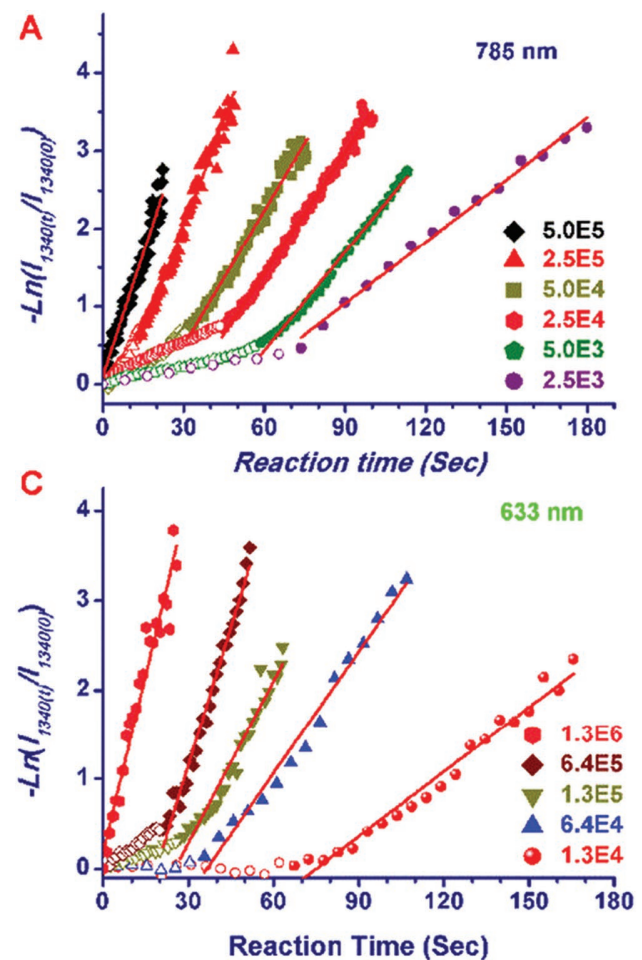
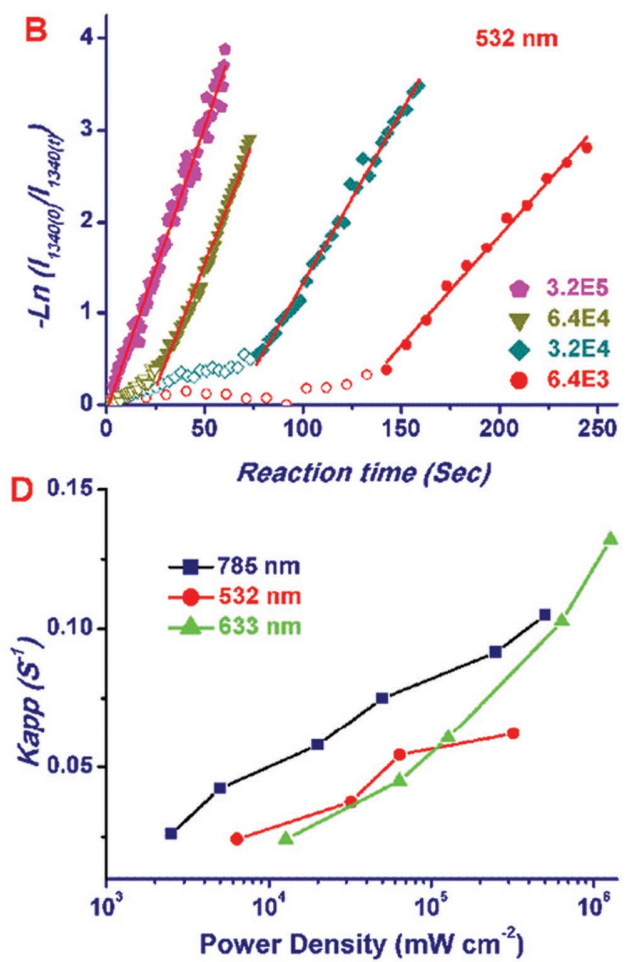

Figure 5. The reaction kinetics of the p-NTP reduction on Ag@Pd (4\%Pd) derived from an in situ SERS study with $785 \mathrm{~nm} \mathrm{A),} 532 \mathrm{~nm}$ B), and $633 \mathrm{~nm}$ C) laser at varying power density, and a plot that summarizes the power density-rate constant relationship D).

633 and $532 \mathrm{~nm}$ wavelength laser was used (Figure 5B,C). However, the rate constant increased only slightly when the $532 \mathrm{~nm}$ laser was employed (Figure 5D). Furthermore, under the excitation of the $532 \mathrm{~nm}$ laser, DMAB was once again produced (Figure S13, Supporting Information). Those DMAB are likely produced from the reductive dimerization of p-NTP, which consumes four electrons. This was reflected in the lowered effectiveness of HEs in catalyzing the production of $\mathrm{p}-\mathrm{ABT}$.

\section{Discussion}

It is generally accepted that upon the excitation of resonant light (or light in the SPR region), partial of the captured photo energy is adsorbed by metal electrons and excited into HEs, meanwhile a portion of the rest energy transforms into heat (PT effect). ${ }^{[4 b]}$ From the SERS trace of the reaction process, especially the 3D graph shown in Figure 3B, it is evident that the whole reaction process was separated into the induction stage and the reduction stage. Since the precondition for the reduction of p-NTP is to approach close enough to active $\mathrm{H}$-species for $-\mathrm{NO}_{2}$, p-NTP molecule should rotate to reach a proper configuration. Meanwhile, as the surface of the $\mathrm{M}(\mathrm{M}=\mathrm{Au}, \mathrm{Ag}) @ \mathrm{Pd}$ surface has been modified with a dense p-NPT monolayer, the diffusion of $\mathrm{BH}_{4}^{-}$to the $\mathrm{Pd}$ catalysis center and activation therein is also a time-consuming process. We believe the speed of these two processes determines the time-scale of the induction period. Considering these two processes are simple physical diffusion/rotation, HEs are less likely to participating in such a process, and we attributed it to the PT effect. In Au@Pd film, for the weak SPR adsorption and their small size, the generated PT is limited. Therefore, it is only illuminated by high power density, $\approx 10 \%$ relative power, can sufficient PT be generated and to allow the reaction to proceed immediately. In alternative, an apparent induction time is needed, and when the PT was totally suppressed by recording the SERS spectra in a placeshift fashion, a long induction time of $600 \mathrm{~s}$ was needed. In the other hand, the strong SPR band at both the Vis and NIR grantees strong light-matter interaction in $\mathrm{Ag} @ \mathrm{Pd}$, and the large size of $\mathrm{Ag}$ means a long path for the traveling of SPR generated HEs to the surface. So larger portion of energy is transferred to $\mathrm{Ag}$ atoms through the frequent inelastic collision between HEs and $\mathrm{Ag}$ lattice atom and transform into $\mathrm{PT}$, which explains the very short induction time in Ag@Pd (Scheme S1, Supporting Information).

Alternatively, the HE is the main driving force for the acceleration of p-NTP reduction itself. The key step for the $\mathrm{p}$-NTP reduction is to break the $\mathrm{N}-\mathrm{O}$ bonds, ${ }^{[25]}$ and converse into p-ABT through intermediates like dihydroxylaminobenzenethiol (DHABT), nitrosobenzenethiol (NSBT), hydroxylaminobenzenethiol (HABT), azoxybenzenethiol (AZOBT), and dimercaptohydroazobenzene. ${ }^{\left[{ }^{[6]}\right.}$ Normally, this process is realized by the active $\mathrm{H}$ species, approximately, $\mathrm{H}-\mathrm{Pd}$, with an activation energy of no more than $100 \mathrm{~kJ} \mathrm{~mol}^{-1}{ }^{[27]}$ However, under the excitation of light, the generated HEs may migrate into the antibond orbit of the nitro groups, and facilitates their cleavage (Scheme S2, Supporting Information). Increasing the power density of SPR light produces more HEs, and if there is no other pathway for the consumption/ waste of these HEs, this increase in the amount of HEs will 
result in an increased reaction rate, or the rate constant will show a linear dependence with the laser power. Indeed, we do observe a high linearly relationship between the power density and the rate constant on Au@Pd (Figure 3D), which is a strong evidence for the HEs promoting nature of this reaction. ${ }^{[9]}$ This is further supported by the wavelengthdependency of the rate constant. Although the energy of $532 \mathrm{~nm}\left(225.0 \mathrm{~kJ} \mathrm{~mol}^{-1}\right), 633 \mathrm{~nm}\left(189.1 \mathrm{~kJ} \mathrm{~mol}^{-1}\right)$, and $785 \mathrm{~nm}$ $\left(152.5 \mathrm{~kJ} \mathrm{~mol}^{-1}\right)$ laser all meet the energy requirement for this process, apparent SPR band of Au@Pd is only observed in the NIR, thus only $785 \mathrm{~nm}$ laser effectively increase the reaction pace of p-NTP reduction. Furthermore, the laser power-independency with the rate constant in $\mathrm{Ag} @ \mathrm{Pd}$, where the existence of PT effect has been demonstrated in the large shortened induce time, further infers PT effect has negligible influence to the NTP reduction process. The absence of SPR acceleration phenomenon points an important fact that there are other routes for the consumption/waste of HEs.

As is revealed in Figure 4, on the Ag@Pd system, the HEs were first consumed by the $\mathrm{O}$ species and $\mathrm{H}^{+}$, and then accelerated the p-NTP to p-ABT transform process, and finally take part in the reductive dimerization of p-NTP (Figure 5, with a $532 \mathrm{~nm}$ laser Scheme S3 in the Supporting Information). We hypothesize that the generated HEs react with the following energy diagram: dimerization of $\mathrm{p}-\mathrm{NTP}>$ reduction of p-NTP by $\mathrm{KBH}_{4}>$ reduction of $\mathrm{H}^{+}$and activation of $\mathrm{O}$ species. ${ }^{[17 \mathrm{e}]}$ In the Au@Pd NWs case, under the excitation of resonant light, c.a. $785 \mathrm{~nm}$ laser, the energy of SPR-generated HEs is insufficient to dimerize p-NTP. Instead, these HEs are only capable of converting p-NTP to p-ABT, and the fast reduction of surface $\mathrm{O}$ species by $\mathrm{KBH}_{4}$ may be the reason why the pathways associated with the activation of $\mathrm{O}$ species was blocked. Therefore, HEs can be effectively channeled into the reduction of p-NTP by $\mathrm{KHB}_{4}$. While when nonresonant 633 or $532 \mathrm{~nm}$ laser light was used, the amount/energy of SPR-generated HEs was limited, thus no apparent photo induced rate enhancement the reaction was observed. In contrary, the Ag@Pd system showed a broad SPR-adsorption band and effectively adsorbed photons with a wavelength of 532, 633, and $785 \mathrm{~nm}$ to excite HEs. However, the majority of the produced HEs were consumed by $\mathrm{O}$ species $/ \mathrm{H}^{+}$, which resulted in no photoacceleration of the p-NTP reduction. Only when most of the $\mathrm{Ag}$ pinholes were blocked by $\mathrm{Pd}$ atoms, can part of the HEs be participated in the reduction of p-NTP. However, under this circumstance, with the increase of photon energy by switch to $532 \mathrm{~nm}$ laser, part of HEs meets the energy requirement for the reductive dimerization of p-NTP, and this resulted in a lower rate enhancement factor.

\section{Conclusion}

In summary, by systematically in situ SERS studying the relationship between rate constant and laser power density for the Pd adatom catalyzed reduction of $\mathrm{p}-\mathrm{NTP}$, we report that the pace of this reaction can be largely enhanced by SPRgenerated HEs. From this unexpected discovery, we proposed an in situ SERS method to track the fate of SPR-generated
HEs. The relative inertness of surface metal and the morphology of catalyst, as well as the relative energy between paralleling reactions were identified as the key factors that influence the utilization of HEs in a given reaction. More specifically, the presence of $\mathrm{Ag}$ pinholes is a key factor for HEs to be leaked to the surrounding ions/molecule. This may also be a pivotal constraint for the usage of $\mathrm{Ag}$ in plasmonic catalysts, especially in reactions that do not require oxygen. The significance of current work lies, first, we conceptual shown the prospect of tracking the important physical-chemical events by quantitative SERS study. Moreover, from the viewpoint of designing Ag-based plasmonic catalyst, besides a few cases where Ag acts alone as the catalyst, ${ }^{[28]} \mathrm{Ag}$ most often forms a bimetal nanocatalyst with another metal like Pd. ${ }^{[29]}$ The waste of HEs by Ag may limit their usage as effective plasmonic catalyst. A plausible solution to this limitation is to replace $\mathrm{Ag} / \mathrm{Pd}$ with $\mathrm{Au} / \mathrm{Pd}$ at the surface. Meanwhile, $\mathrm{Ag}$ / $\mathrm{AgX},{ }^{[30]}$ a promising visible-light photocatalyst in which $\mathrm{Ag}$ adsorbs light and transfers the generated hole rather than the HEs to AgX. The self-consumption of HEs reveal in this study may be very meaningful for the lasting recruitment of holes, and maybe an explanation for the good stability of $\mathrm{Ag} / \mathrm{AgX}$.

\section{Experimental Section}

Fabrication of Au@Pd and Au@Ag@Pd Ultrathin Nanowire Film: The ultrathin Au@Pd NWs were synthesized according to our recent developed procedure (unpublished result). Briefly, under vigorous stirring, $3 \mathrm{~mL}$ fresh prepared $\mathrm{KBH}_{4}$ solution was quickly injected into a $47 \mathrm{~mL}$ ice-cooled aqueous solution containing $0.05 \mathrm{mmol}$ of $\mathrm{HAuCl}_{4}$ and $25 \mathrm{mg}$ of TX-114, and the resulting solution was placed in a septum-sealed $50 \mathrm{~mL}$ flask. After the addition of $\mathrm{KBH} 4$, the mixture was further stirred for $1 \mathrm{~min}$ at $0{ }^{\circ} \mathrm{C}$. For the preparation of Au@Pd core-shell NWs, $10.0 \mathrm{~mL}$ fresh-synthesized $\mathrm{Au}$ NWs dispersion was transferred to new flasks, and then $0.75 \mathrm{~mL}$ of ice-cold $1.0 \times 10^{-3} \mathrm{M} \mathrm{Na}_{2} \mathrm{PdCl}_{4}$ solution was added into the flask dropwise under magnetic stirring, the coverage of $\mathrm{Pd}$ is 1/4/monolayer. While in the case of Au@Ag@Pd NWs, $3.0 \mathrm{~mL}$ $1.0 \times 10^{-3} \mathrm{M} \mathrm{AgNO}_{3}$ was added into $10.0 \mathrm{~mL}$ fresh-synthesized $\mathrm{Au}$ NWs dispersion under magnetic stirring, followed by adding 0.75 , $1.50 \mathrm{~mL}$ of ice-cold $1.0 \times 10^{-3} \mathrm{M} \mathrm{Na}_{2} \mathrm{PdCl}_{4}$ solution.

To deposit the thin film of Au@Pd or Au@Ag@Pd NWs on the $\mathrm{SiO}_{2}$ wafer, 1.935 or 2.475 mL of Au@Pd, or Au@Ag@Pd dispersion was added to the well of a 24-well plate, respectively, where a $1.0 \mathrm{~cm} \times 1.0 \mathrm{~cm} \mathrm{SiO}$, slide is already placed. To accelerate the deposition process, $5 \times 10^{-3} \mathrm{M} \mathrm{NaCl}$ was added into the Au@Pd NWs dispersion, but no $\mathrm{NaCl}$ was needed for Au@Ag@Pd. After mixing, the plate was placed undisturbed at $4^{\circ} \mathrm{C}$. After $12 \mathrm{~h}$, the colorless upper solution was carefully removed by pipette, and the black deposited layer left was slowly dried in a refrigerator and then washed with cold ethanol.

Preparation of Ag@Pd NPFs: To make Ag NPF catalytic active and Pd SERS active, Pd nanocatalyst, both in the form of Pd overlayer and Pd branches, was in situ synthesized and cosediment with Ag NWs that synthesized with a similar method to that of $\mathrm{Au}$ NWs. Specifically, after the injection of $12 \times 10^{-3} \mathrm{M} \mathrm{KBH}_{4}$ into $2.0 \times 10^{-3} \mathrm{M} \mathrm{AgNO}_{3}$ solution, desired amount of $1 \times 10^{-3} \mathrm{M}$ 
$\mathrm{Na}_{2} \mathrm{PdCl}_{4}$ was dropwisely added the solution under vigorous stirring. ${ }^{[21]}$ Again, the synthesized hybrid nanostructure was transferred into a 24-well plate, where a $250 \mu \mathrm{m}$ transparent $\mathrm{SiO}_{2}$ wafer was already placed in. The container was stored undisturbed at $4{ }^{\circ} \mathrm{C}$ for $8 \mathrm{~h}$. After that, the upper colorless aqueous solution was removed carefully with a pipette, and the formed black gel-like thin layer was dried at $4{ }^{\circ} \mathrm{C}$ and $70 \%$ relative moisture and washed twice with cold ethanol.

Operando SERS Monitoring of Catalyzed Reaction: To in situ monitoring the Pd catalyzed $\mathrm{KBH}_{4}$ reduction of $\mathrm{p}-\mathrm{NTP}$ to $\mathrm{p}-\mathrm{ABT}$, the deposited NW film and Ag@Pd NPF were immersed in $0.1 \times 10^{-3} \mathrm{M}$ p-NTP ethanol solution for $30 \mathrm{~min}$ to form a SAM. After washed by copious of ethanol and air-dried, the slide was stuck onto a labmade reactor, with NWs/NPF film exposed to the interior of the reactor. The reactor was placed on the XYZ stage of the Raman microscope. The Raman spectra was acquired in successive mode from the film, and after a stable spectrum was acquired, desired concentration of $\mathrm{KBH}_{4}$ aqueous solution was injected the reactor to initiate the reaction. The reaction rate constant $K$ can be calculated by equation

$$
\ln C_{t} / C_{0}=\ln \left(I_{1340(t)} / I_{1340(t=0))}=k t+b\right.
$$

All Raman experiments were performed on a Renishaw InVia Raman microscope equipped with 532, 633, and $785 \mathrm{~nm}$ Laser lines as the excitation source, and the Raman band of a silicon wafer at $520 \mathrm{~cm}^{-1}$ was used to calibrate the spectrometer. The power attenuated to $0.0001 \%, 0.05 \%, 0.1 \%, 0.5 \%, 1.0 \%, 5 \%$, and $10 \%$ of the $785 \mathrm{~nm}$ laser (about $100 \mathrm{~mW}$ at sample), $0.1 \%$, $0.5 \%, 1.0 \%, 5 \%$, and $10 \%$ of the $633 \mathrm{~nm}(10 \mathrm{~mW}) / 532 \mathrm{~nm}$ laser $(5 \mathrm{~mW})$.

\section{Supporting Information}

Supporting Information is available from the Wiley Online Library or from the author.

\section{Acknowledgements}

The authors acknowledge the anonymous reviewers for taking time and effort in evaluating/improving our manuscript. This work was financially supported by the Chinese Academy of Sciences (XDB14020101), Ministry of Science and Technology (2014CB932000), and the National Natural Science Foundation of China (21577157, 21337004 and 21321004).

[1] N. S. Lewis, D. G. Nocera, Proc. Natl. Acad. Sci. USA 2006, 103, 15729.

[2] X. G. Zhu, S. P. Long, D. R. Ort, Curr. Opin. Biotechnol. 2008, 19, 153.

[3] N. S. Lewis, Science 2007, 315, 798.

[4] a) S. Linic, P. Christopher, D. B. Ingram, Nat. Mater. 2011, 10, 911; b) M. J. Kale, T. Avanesian, P. Christopher, ACS Catal. 2014, 4, 116; c) C. L. Wang, D. Astruc, Chem. Soc. Rev. 2014, 43, 7188; d) N. E. Motl, A. F. Smith, C. J. DeSantis, S. E. Skrabalak, Chem. Soc. Rev. 2014, 43, 3823.

[5] a) W. Xie, S. Schlucker, Nat. Commun. 2015, 6, 7570; b) H. de Puig, J. O. Tam, C. W. Yen, L. Gehrke, K. Hamad-Schifferli, J. Phys. Chem. C 2015, 119, 17408.

[6] P. K. Jain, K. S. Lee, I. H. El-Sayed, M. A. El-Sayed, J. Phys. Chem. B 2006, 110, 7238.

[7] a) H. Ghasemi, G. Ni, A. M. Marconnet, J. Loomis, S. Yerci, N. Miljkovic, G. Chen, Nat. Commun. 2014, 5, 4449; b) J. B. Song, X. Y. Yang, O. Jacobson, L. S. Lin, P. Huang, G. Niu, Q. J. Ma, X. Y. Chen, ACS Nano 2015, 9, 9199; c) N. J. Hogan, A. S. Urban, C. Ayala-Orozco, A. Pimpinelli, P. Nordlander, N. J. Halas, Nano Lett. 2014, 14, 4640.

[8] W. B. Hou, W. H. Hung, P. Pavaskar, A. Goeppert, M. Aykol, S. B. Cronin, ACS Catal. 2011, 1, 929.

[9] P. Christopher, H. L. Xin, S. Linic, Nat. Chem. 2011, 3, 467.

[10] a) M. Murdoch, G. I. N. Waterhouse, M. A. Nadeem, J. B. Metson, M. A. Keane, R. F. Howe, J. Llorca, H. Idriss, Nat. Chem. 2011, 3, 489; b) Z. K. Zheng, T. Tachikawa, T. Majima, J. Am. Chem. Soc. 2015, 137, 948; c) P. Zhang, T. Wang, J. L. Gong, Adv. Mater. 2015, 27, 5328

[11] a) X. Q. Huang, Y. J. Li, Y. Chen, H. L. Zhou, X. F. Duan, Y. Huang, Angew. Chem. Int. Ed. 2013, 52, 6063; b) F. Wang, C. H. Li, H. J. Chen, R. B. Jiang, L. D. Sun, Q. Li, J. F. Wang, J. C. Yu, C. H. Yan, J. Am. Chem. Soc. 2013, 135, 5588.

[12] S. Mukherjee, F. Libisch, N. Large, O. Neumann, L. V. Brown, J. Cheng, J. B. Lassiter, E. A. Carter, P. Nordlander, N. J. Halas, Nano Lett. 2013, 13, 240.

[13] A. Marimuthu, J. W. Zhang, S. Linic, Science 2013, 339, 1590.

[14] a) Y. F. Huang, M. Zhang, L. B. Zhao, J. M. Feng, D. Y. Wu, B. Ren, Z. Q. Tian, Angew. Chem. Int. Ed. 2014, 53, 2353; b) M. T. Sun, H. X. Xu, Small 2012, 8, 2777.

[15] a) K. L. Kelly, E. Coronado, L. L. Zhao, G. C. Schatz, J. Phys. Chem. B 2003, 107, 668; b) T. R. Jensen, M. D. Malinsky, C. L. Haynes, R. P. Van Duyne, J. Phys. Chem. B 2000, 104, 10549.

[16] a) J. T. Li, S. K. Cushing, J. Bright, F. K. Meng, T. R. Senty, P. Zheng, A. D. Bristow, N. Q. Wu, ACS Catal. 2013, 3, 47; b) K. lizuka, T. Wato, Y. Miseki, K. Saito, A. Kudo, J. Am. Chem. Soc. 2011, 133, 20863; c) D. B. Ingram, S. Linic, J. Am. Chem. Soc. 2011, 133, 5202 .

[17] a) R. Liu, J. F. Liu, Z. M. Zhang, L. Q. Zhang, J. F. Sun, M. T. Sun, G. B. Jiang, J. Phys. Chem. Lett. 2014, 5, 969; b) V. Joseph, C. Engelbrekt, J. D. Zhang, U. Gernert, J. Ulstrup, J. Kneipp, Angew. Chem. Int. Ed. 2012, 51, 7592; c) W. Xie, C. Herrmann, K. Kompe, M. Haase, S. Schlucker, J. Am. Chem. Soc. 2011, 133, 19302; d) X. J. Chen, G. Cabello, D. Y. Wu, Z. Q. Tian, J. Photochem. Photobiol. C 2014, 21, 54; e) X. Zhang, P. J. Wang, Z. L. Zhang, Y. R. Fang, M. T. Sun, Sci. Rep. 2014, 4, 5407; f) J. L. Wang, R. A. Ando, P. H. C. Camargo, Angew. Chem. Int. Ed. 2015, 54, 6909; g) T. Hartman, C. S. Wondergem, K. N. A. van den Berg, B. M. Weckhuysen, J. Phys. Chem. Lett. 2016, 7, 1570.

[18] a) Z. L. Zhang, P. Xu, X. Z. Yang, W. J. Liang, M. T. Sun, J. Photochem. Photobiol. C 2016, 27, 100; b) Y. Fang, Z. Zhang, M. Sun, Rev. Sci. Instrum. 2016, 87, 033104.

[19] J. F. Huang, Y. H. Zhu, M. Lin, Q. X. Wang, L. Zhao, Y. Yang, K. X. Yao, Y. Han, J. Am. Chem. Soc. 2013, 135, 8552.

[20] Q. F. Zhang, D. A. Blom, H. Wang, Chem. Mater. 2014, 26, 5131.

[21] R. Liu, J. F. Sun, D. Cao, L. Q. Zhang, J. F. Liu, G. B. Jiang, Chem. Commun. 2015, 51, 1309.

[22] a) B. Dong, Y. Fang, L. Xia, H. Xu, M. Sun, J. Raman Spectrosc. 2011, 42, 1205; b) H. Yingzhou, F. Yurui, Y. Zhilin, S. Mengtao, J. Phys. Chem. C 2010, 114, 18263.

[23] M. Sun, Z. Zhang, Z. H. Kim, H. Zheng, H. Xu, Chem. Eur. J. 2013, $19,14958$.

[24] M. Sun, Y. Huang, L. Xia, X. Chen, H. Xu, J. Phys. Chem. C 2011, 115, 9629.

[25] H. Y. Zhu, X. B. Ke, X. Z. Yang, S. Sarina, H. W. Liu, Angew. Chem. Int. Ed. 2010, 49, 9657. 
[26] H. K. Choi, W. H. Park, C. G. Park, H. H. Shin, K. S. Lee, Z. H. Kim, J. Am. Chem. Soc. 2016, 138, 4673.

[27] K. Zhang, J. J. Zhao, J. Ji, Y. X. Li, B. H. Liu, Anal. Chem. 2015, 87, 8702.

[28] Y. Lei, F. Mehmood, S. Lee, J. Greeley, B. Lee, S. Seifert, R. E. Winans, J. W. Elam, R. J. Meyer, P. C. Redfern, D. Teschner, R. Schlogl, M. J. Pellin, L. A. Curtiss, S. Vajda, Science 2010, 328, 224.

[29] a) G. X. Pei, X. Y. Liu, A. Q. Wang, A. F. Lee, M. A. Isaacs, L. Li, X. L. Pan, X. F. Yang, X. D. Wang, Z. J. Tai, K. Wilson, T. Zhang,
ACS Catal. 2015, 5, 3717; b) H. P. Rong, S. F. Cai, Z. Q. Niu, Y. D. Li, ACS Catal. 2013, 3, 1560; c) D. A. Slanac, W. G. Hardin, K. P. Johnston, K. J. Stevenson, J. Am. Chem. Soc. 2012, 134, 9812.

[30] P. Wang, B. B. Huang, X. Y. Qin, X. Y. Zhang, Y. Dai, J. Y. Wei, M. H. Whangbo, Angew. Chem. Int. Ed. 2008, 47, 7931.

Received: May 26, 2016

Revised: August 23, 2016

Published online: September 27, 2016 